\title{
Cross-modal Correspondences Enhance Performance on a Colour-to- Sound Sensory Substitution Device
}

\author{
Giles Hamilton-Fletcher*, Thomas D. Wright and Jamie Ward
}

School of Psychology and Sackler Centre for Consciousness Science, University of Sussex, UK

\section{Supplementary Figure S1}

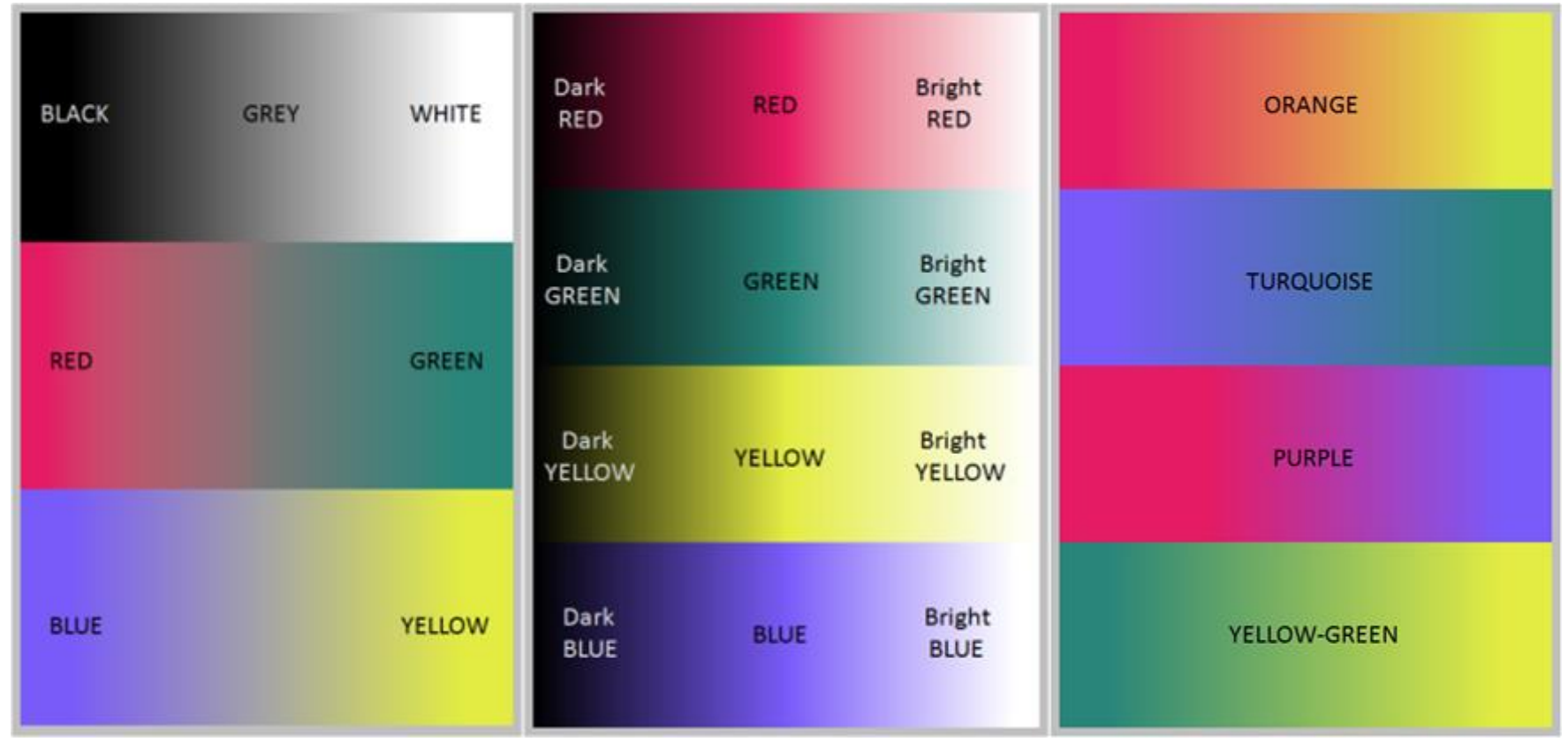

Figure S1. Creole colour training materials, left image allows users to explore the main seven colours, middle image allows users to explore bright and dark variants of the four unique hues, and right allows users to explore complicated colour combinations when two unique hues mix.

A demonstration of the Creole can be found at the following link:

https://www.youtube.com/watch?v=vplaOczMQBo 